Published in final edited form as:

J Pain. 2013 June ; 14(6): 579-589. doi:10.1016/j.jpain.2013.01.767.

\title{
Augmented Central Pain Processing in Vulvodynia
}

\author{
Johnson P. Hampson ${ }^{\mathrm{a},{ }^{,}, \text {Barbara D. Reed }}{ }^{\mathrm{b}}$, Daniel J. Clauw ${ }^{\mathrm{a}, \mathrm{c}}$, Rupal Bhavsard, Richard H. \\ Gracely $^{\mathrm{e}}$, Hope K. Haefner ${ }^{\dagger}$, and Richard E. Harris ${ }^{\mathrm{a}, \mathrm{c}}$ \\ ${ }^{a}$ Chronic Pain and Fatigue Research Center, Department of Anesthesiology, University of \\ Michigan, 24 Frank Lloyd Wright Drive, P.O. Box 385, Lobby M, Ann Arbor, MI 48106 \\ ${ }^{b}$ Department of Family Medicine, University of Michigan, Ann Arbor, MI \\ 'Department of Internal Medicine-Rheumatology, University of Michigan, Ann Arbor, MI \\ dNeurology Department, University of Pennsylvania, Philadephia, PA, USA \\ eDepartment of Endodontics, The University of North Carolina, Chapel Hill, NC \\ fDepartment of Obstetrics and Gynecology, University of Michigan, Ann Arbor, MI
}

\begin{abstract}
Vulvodynia (VVD) is a chronic pain disorder, wherein women display sensitivity to evoked stimuli at the vulva and/or spontaneous vulvar pain. Our previous work suggests generalized hyperalgesia in this population, however little is known about central neurobiological factors that may influence pain in VVD. Here we investigated local (vulvar) and remote (thumb) pressure evoked pain processing in 24 VVD patients compared to 13 age-matched, pain-free healthy controls (HC). As a positive control we also examined thumb pressure pain in 24 fibromyalgia (FM) patients. The VVD and FM patients displayed overlapping insular brain activations that were greater than $\mathrm{HC}$, in response to thumb stimulation ( $\mathrm{P}<0.005$ corrected). Compared to $\mathrm{HC}$, VVD participants displayed greater levels of activation during thumb stimulation within the insula, dorsal mid-cingulate, posterior cingulate and thalamus $(P<0.005$ corrected). Significant differences between VVD subgroups (primary versus secondary and provoked versus unprovoked) were seen within the posterior cingulate with thumb stimulation, and within the precuneus region with vulvar stimulation (provoked versus unprovoked only). The augmented brain activation in VVD patients in response to a stimulus remote from the vulva suggests central neural pathology in this disorder. Moreover, differing central activity between VVD subgroups suggests heterogeneous pathologies within this diagnosis.
\end{abstract}

\section{Keywords}

vulvodynia (VVD); fibromyalgia (FM); healthy controls (HC); functional magnetic resonance imaging (fMRI); central nervous system (CNS)

\footnotetext{
(C) 2013 The American Pain Society. Published by Elsevier Inc. All rights reserved.

*Corresponding author: Johnson P. Hampson, M.S.B.M.E, Chronic Pain and Fatigue Research Center, Department of Anesthesiology, 24 Frank Lloyd Wright Drive, P.O. Box 385, Lobby M, Ann Arbor, MI 48106. Phone: (734) 998 - 7019. Fax: (734) 998 - 6900. jhampson@med.umich.edu.

Publisher's Disclaimer: This is a PDF file of an unedited manuscript that has been accepted for publication. As a service to our customers we are providing this early version of the manuscript. The manuscript will undergo copyediting, typesetting, and review of the resulting proof before it is published in its final citable form. Please note that during the production process errors may be discovered which could affect the content, and all legal disclaimers that apply to the journal pertain.

Disclosures:

The authors declare that they have no conflict of interest.
} 


\section{Introduction}

Vulvodynia (VVD), a chronic pain condition associated with painful intercourse, tampon use and/or spontaneous vulvar pain, occurs in approximately 7-10\% of women in the United States, ${ }^{19,30}$ yet little is known about its pathophysiology or etiology.${ }^{18}$ Previous clinical studies suggest that women with VVD have lower pain thresholds (i.e. hyperalgesia) in the vulvar region, and also in peripheral regions such as the thumb, deltoid, and shin (i.e. generalized hyperalgesia). ${ }^{13,14}$ This global decrease in pain thresholds suggests that factors remote from the vulva, potentially the central nervous system, may play a role in symptom expression in these women.

One way of looking into central mechanisms involved in human pain processing is by using functional magnetic resonance imaging (fMRI) in conjunction with evoked pain stimuli. In typical evoked pain fMRI studies, changes in the blood oxygenation level dependent (BOLD) effect are observed during painful thermal, electrical and pressure stimulation. Multiple fMRI studies of pain have indicated a constellation of brain regions activated during pain. These regions included the insula, the somatosensory cortex, the amygdala, the dorsolateral prefrontal cortex, the thalamus, and the anterior and posterior cingulate. ${ }^{8,22,34}$ Emerging data suggests that pain disorders that have previously been thought to be more peripheral in nature, such as chronic low back pain ${ }^{15}$ and osteoarthritis ${ }^{18}$, also show enhanced brain activity in response to pain, thus highlighting the potential role of the brain in chronic pain.

In the only previous fMRI study in VVD, significant cortical activation in response to experimental vulvar pressure pain was observed for patients in regions such as the insula, somatosensory and premotor cortex. ${ }^{29}$ Here we explore the brain response resulting from experimental pressure pain stimuli applied to a region distant from the vulva (i.e. the thumb) in women with VVD as compared to two age- and sex-matched groups: Fibromyalgia (FM) patients serving as our positive control, and pain free healthy control (HC) participants as our negative control.

FM is a functional chronic pain condition associated with altered brain structure, function, and neurochemistry. ${ }^{23,21}$ Findings of generalized hyperalgesia (an increased response to painful stimuli) and allodynia (painful response to a non-painful stimuli) are also present in fibromyalgia. ${ }^{14,17}$ We hypothesized that the enhanced brain response seen in women with FM compared to HCs in pain related regions such as the insula cortex, somatosensory, dorsolateral prefrontal cortex, thalamus and cingulate cortex, ${ }^{17,9}$ may also be present in individuals with VVD, if these two pain conditions have similar pathobiology, as suggested by the observed generalized hyperalgesia. Further, as an exploratory analysis we sought to identify brain activation differences between previously suggested clinical VVD subgroups such as those with primary or secondary VVD, ${ }^{2,24}$ provoked or unprovoked, ${ }^{24}$ and generalized or localized pain. ${ }^{26}$ Finally, in addition to thumb pressure stimuli, we also compared brain response to vulvar stimuli, among VVD patients and HCs, in an effort to replicate previous work. ${ }^{29}$

\section{Methods}

\subsection{Participants}

VVD and pain free healthy controls (HCs)-The study was approved by the University of Michigan Medical School Institutional Review Board and all participants gave written consent to be in this study. VVD patients were recruited at two sites: the Vulva Clinic at the University of Michigan Family Practice Center at Chelsea or the University of Michigan Center for Vulvar Diseases. Patients who were given the diagnosis of VVD at 
their initial evaluation were invited to participate at that time. VVD patient inclusion criteria were women age 18-60 years with pain at the opening of the vagina, present for a minimum of 3 months, with no other vulvar disorder that would explain the pain. The participants were asked to fill out a detailed self-administered questionnaire asking about the history of their symptoms and the characteristics of their pain to determine their subgroup category. Patients included were categorized into subtypes of: primary VVD (present since first intercourse or tampon use) versus secondary VVD (occurring after a pain-free period of time); provoked pain (in response to intercourse, tampon use, or touch) versus unprovoked pain (present without provocation); and those whose pain was localized (to the introitus only) or more generalized (to more peripheral aspects of the vulva). Inclusion criteria for HCs were women presenting for a routine gynecological examination at one of these University of Michigan clinics who did not have current vulvar pain and who did not have a history of vulvar pain that had lasted for more than three months. Exclusion criteria for HCs were women that were currently pregnant, nursing, or within 6 months postpartum, had a diagnosis of FM or chronic fatigue syndrome, had a definitive vulvar rash or lesion, or had a history of vulvar laser surgery, past vestibulectomy, or an anterior and/or posterior repair. All VVD and HCs underwent a genital examination to confirm their case or control status. This exam included visual inspection, Q-tip testing at $27 \mathrm{vulvar} / \mathrm{hymenal}$ sites, and a vaginal inspection. An in-office examination of vaginal secretions was conducted (ph, whiff test, normal saline and $\mathrm{KOH}$ microscopic examinations), and a vaginal yeast culture was also obtained. See Supplementary Table 1 for participant demographics data.

FM participants (positive controls) - The data on FM participants used in this analysis were obtained from a similar study also performed at the University of Michigan. ${ }^{21}$ All FM participants met the American College of Rheumatology (ACR) 1990 criteria for classification of fibromyalgia. FM subjects followed similar participant inclusion-exclusion criteria and had identical imaging parameters for the fMRI scan as did the VVD participants and HCs (see below). The exclusion and inclusion criteria for FM participants have been reported previously. ${ }^{21}$ All participants, including VVD, FM and HCs, were right handed.

\subsection{Clinical and Experimental Pain}

VVD and healthy control participants were evaluated for pressure sensitivity at both the thumb and vulva, as previously described. ${ }^{28,14} \mathrm{FM}$ positive controls underwent thumb pressure pain sensitivity testing only. In brief, for thumb pressure stimuli the stimulator consists of a $1 \mathrm{~cm}^{2}$ hard rubber circular probe supported by a plastic housing. Ascending pressures were first administered to the thumb using a hydraulic system activated by calibrated weights placed on a moveable table. Vulvar pressure stimuli was applied via a vulvodolorimeter consisting of low resistance syringes connected by a 3-way valve that allowed measurement of pressures applied to a cotton swab attached to the device. ${ }^{14}$ Pressures were increased gradually at a rate of $1 \mathrm{~kg} / \mathrm{s}$ for thumb and $0.1 \mathrm{~kg} / \mathrm{s}$ for vulvar stimuli, to a maximum pressure of $12 \mathrm{~kg} / \mathrm{cm}^{2}$ for thumb and $1.86 \mathrm{~kg} / \mathrm{cm}^{2}$ for vulva or until the participant reached their pain tolerance level. Subjects were instructed to indicate when they first perceived pain and their pain ratings were recorded based on the Gracely Box Scale (GBS; 0-20 scale). ${ }^{28}$ Thumb pressure sensitivity was additionally assessed via application of a series of random pressures that were applied using the multiple random staircase (MRS) method. ${ }^{16}$ These values of pressure sensitivity, determined during the ascending pressure assessment, were used to determine three independent pressure staircases that are titrated (via in-house computer software) to produce pain sensation ratings for "faint" pain (1 on the GBS), "mild" pain (8 on the GBS) and "slightly intense" pain (13.5 on the GBS). Prior to scanning, each participant's clinical pain experience was also assessed using the short-form of McGill Pain Questionnaire (SF-MPQ). ${ }^{25}$ The main component of 
the SF-MPQ consist of 15 pain descriptors (11 sensory and 4 affective) ranked on an intensity scale $0=$ none, $1=$ mild, $2=$ moderate, $3=$ severe.

\subsection{Evoked pain during fMRI}

As menstrual cycle status is known to influence pain processing, all pre-menopausal women were scanned only on days 5-14 of their menstrual cycle. ${ }^{32,1}$ During the fMRI, separate runs for pain activations were conducted for the thumb pressure stimulation and for the vulvar pressure stimulation. Each run was divided into thirteen $25 \mathrm{sec}$ OFF periods and twelve $25 \mathrm{sec}$ ON periods for both thumb and vulvar pressure stimuli. The twelve ON periods consisted of application of three "innocuous touch" (0 on the GBS), three "faint", three "mild", and three "slightly intense" pain pressures (see above) presented in a pseudorandom order that was identical for all participants.

During thumb stimulation, pressures were applied discretely to the left thumb using an fMRI compatible remote stimulation device. ${ }^{28}$ In brief, a $1 \mathrm{~cm}^{2}$ rubber probe was positioned over the thumb by a plastic housing and the system was activated by a pneumatic system, developed internally and driven by Agilent-Vee software (Santa Clara, CA), that regulated the amount and timing of stimulus pressure generated. Vulvar stimulation was applied using a vulvodolorimeter ${ }^{14}$ that was modified for fMRI. This was programmed to produce remote pressures determined in their experimental pain assessment session outside the scanner, ranging from non-painful to painful levels. The vulvodolorimeter consisted of an air piston that drove a cotton swab which provided steady pressure to the introitus at the 5 o'clock position lateral to the hymenal ring. This pressure was then actively released by negative pressure to the piston.

The fMRI scans were done within a week of each participant's clinical pain assessment. Also at the end of each fMRI experiment the subjects were asked to rate their lowest and their highest pain experienced during the scan using the GBS.

\subsection{Functional MRI data acquisition and analysis}

Functional MRI scans were performed using a 3 Tesla General Electric scanner (Signa LX, Milwaukee, WI). For each participant the anatomical overlay scans were determined using a T1-weighted gradient echo data set (TR $225 \mathrm{~ms}$, TE $3.9 \mathrm{~ms}$, flip angle $90^{\circ}$, FOV $22 \mathrm{~cm}$, number of slices 48 , slice thickness $3.0 \mathrm{~mm}$ ), and the functional scans were acquired using multi-slice, forward spiral fMRI acquisition (TR $2500 \mathrm{~ms}$, TE 30ms, flip angle $90^{\circ}$, FOV $22 \mathrm{~cm}$, number of slices 48 , slice thickness $3.0 \mathrm{~mm}$, voxel size $3.44 \times 3.44 \times 3$ ) and slice-time corrected. The scans covered the whole brain from the top of the cortex to the base of the cerebellum. During each functional run the data from the whole brain was acquired in 256 volumes. The first six volumes in each run were discarded to allow for T1 equilibration effects.

Preprocessing of fMRI images were performed using the SPM5 software package (Statistical Parametric Mapping; Wellcome Department of Cognitive Neurology, London, UK) running under Matlab7.5. Preprocessing steps included re-angulation of images to center at the anterior commissure, realignment of all images to the first volume of each run to correct for intra-scan movements, co-registration to the T1 anatomical image, spatial normalization to the MNI (Montreal Neurological Institute) space, and smoothing with a Gaussian kernel of 8mm FWHM (Full Width at Half Maximum) was used to compensate for small residual anatomic variations across participants. A general linear model was constructed with parameters corresponding to the particular levels of pressure applied in each block to the thumb or the vulva, which were analyzed separately. Parameter estimates of block-related activity were established for each voxel, and contrast images were 
calculated by applying linear contrasts to the parameter estimates of each block to produce statistical parametric maps. Our analyses was performed on the brain volumes collected during contrast conditions of "slightly intense" pain (13.5 GBS) versus the "innocuous touch" condition for pressures applied either to the thumb or the vulva. The results obtained for whole brain analysis, were thresholded at an uncorrected $P$ value of $<0.001$ and significant clusters were identified at $P$ value $<0.05$ family-wise-error corrected. ${ }^{35} \mathrm{We}$ also used small volume correction (SVC) with a spherical search volume of $5 \mathrm{~mm}$ radius placed on the resultant pain regions of interest (ROI) using coordinates obtained from previous $\mathrm{FM}^{17,9}$ and $\mathrm{VVD}^{29} \mathrm{fMRI}$ studies (see below Analysis 3 ). Significant findings using SVC were reported in ROIs of $P<0.05$ corrected. Identified ROIs were extracted using the Marsbar toolbox ${ }^{3}$ and exported to SPSS (Statistical Analysis Software Statistics 19, Chicago, IL), to explore for outliers and to examine individual responses to pressure pain, and to correlate with clinical pain ratings. Image acquisition and image processing followed identical methodology for all participants. All participants completing the study received paid compensation.

Analysis 1 -- Comparison of VVD, FM and HCs-A whole brain two sample t-test analysis was done comparing both the pain groups (VVD+FM) versus HCs to identify regional activations across the pain groups that differed from healthy controls. This analysis was performed for thumb pressure stimuli runs as FM participants did not receive vulvar testing.

Analysis 2 -- Comparison of VVD participants versus HCs-VVD and HC were analyzed in whole brain analyses separately for thumb and vulvar pressure stimuli.

Analysis 3 -- Region of interest (ROI) analysis-Region of interest analysis was done using coordinates obtained from previous $\mathrm{FM}^{17}$ and $\mathrm{VVD}^{29}$ studies for regions displaying differences between cases and controls, including the anterior insula, mid insula, inferior parietal lobule, amygdala, premotor cortex, primary and secondary somatosensory cortex. For these regions group difference t-test analyses were also done comparing VVD patients and HCs for both thumb and vulvar pressure stimuli. Correlation analysis comparing clinical pain scores from SF-MPQ and extracted percent BOLD activation patterns was performed for these ROIs.

Analysis 4 -- Comparison of proposed subgroups of VVD cases-Regions identified as having significantly more activity in women with VVD, as compared to HC (from Analysis 2 above), were tested using an independent sample t-test comparison of participants meeting criteria for previously proposed VVD subgroupings. These included those with primary symptoms versus secondary; those with provoked-pain only versus those who also had unprovoked pain; and those with generalized versus localized pain. As an additional approach to investigating the neural response in VVD subgroups, the pain activated whole brain response during the vulvar pressure pain stimulation was also assessed for paired comparisons of subgroups, using a two sample t-test analysis.

\section{Results}

\subsection{Study enrollment and exclusions}

Forty-four participants qualifying for the fMRI scan (based on diagnosis, and age in the case of healthy control women) were enrolled in the study of VVD cases $(n=25)$ versus healthy controls $(\mathrm{n}=19)$. As expected, there was no significant difference in ages across groups $(P=0.61)$. Cases and controls did not differ in height $(\mathrm{p}=0.82)$ and weight $(\mathrm{p}=0.18)$, and the physical examinations confirmed the lack of dermatologic disorders. No differences between 
cases and controls were found in vaginal or cervical discharge, although cases were more likely to have any erythema than were controls $(27 \%$ vs $0 \%$, p < 0.001$)$. Cases were more sensitive to q-tip pressure at the introitus and hymenal remnants than were controls $(\mathrm{p}<0.001)$. In-office laboratory assessment indicated no positive whiff test or clue cells in cases or controls, and the vaginal $\mathrm{pHs}$ and background flora appearances were similar, although cases were more likely to have $>10 \mathrm{WBCs} / \mathrm{hpf}$ ( $5.4 \%$ vs. $0.0 \%$ respectively, $\mathrm{p}=0.04$ ). Candida species were found on culture in $16.2 \%$ of cases and $11.6 \%$ of controls $(\mathrm{p}=0.55)$, and treatment occurred prior to fMRI testing.

FMRI data on six (1 VVD \& $5 \mathrm{HCs}$ ) participants were excluded due to poor image quality, and data from one $\mathrm{HC}$ was excluded from the analysis due to excessive head motion (greater than $2 \mathrm{~mm}$ ) inside the scanner. The final participants for thumb pressure stimuli analysis were twenty-four women with VVD (mean age \pm SD: $31.88 \pm 10.3$ years), thirteen HCs (mean age \pm SD: $35.36 \pm 10.3$ years) and twenty-four female FM participants (age matched to those with VVD, mean age \pm SD of $33.5 \pm 10.2$ years). Although the level of pressure used to stimulate the vulva was individually determined during the psychophysical testing in the office, $5 \mathrm{VVD}$ participants and $5 \mathrm{HCs}$ reported little or no vulvar pain (rating less than 5 in a Gracely box scale) during their fMRI vulvar run. For this reason these 10 participants were excluded from the analysis involving vulvar pressure stimulation. These exclusions resulted in the number of final participants for the vulvar pressure analysis being nineteen VVD and eight HCs (Figure 1).

\subsection{VVD and FM participants display enhanced pressure pain sensitivity}

The mean vulva pressure pain thresholds measured in the clinical evaluation prior to fMRI testing were significantly lower for VVD participants compared to HCs (see figure 2a): faint pain (Mean \pm SD VVD: $0.06 \pm 0.05 \mathrm{~kg}$, HCs: $0.29 \pm 0.16 \mathrm{~kg}, P=0.00001$ ), mild pain (Mean \pm SD VVD: $0.07 \pm 0.09 \mathrm{~kg}, \mathrm{HCs}: 0.38 \pm 0.18 \mathrm{~kg}, P=0.00001$ ) and slightly intense pain (Mean $\pm \mathrm{SD}$ VVD: $0.31 \pm 0.22 \mathrm{~kg}$, HCs: $0.63 \pm 0.43 \mathrm{~kg}, P=0.01$ ). The mean thumb pressure pain thresholds were also significantly lower for VVD participants compared to HCs (see figure $2 \mathrm{~b}$ ): mild pain stimuli (Mean \pm SD VVD: $2.50 \pm 1.32 \mathrm{~kg}$, HCs: $3.64 \pm 2.63 \mathrm{~kg}, P=0.01$ ) and a trend towards lower pressure pain threshold values for faint pain (Mean \pm SD VVD: $0.81 \pm 0.70 \mathrm{~kg}$, HCs: $1.12 \pm 1.59 \mathrm{~kg}, P=0.10$ ) and slightly intense pain (Mean \pm SD VVD: $4.62 \pm 1.62 \mathrm{~kg}$, HCs: $5.78 \pm 2.28 \mathrm{~kg}, P=0.10$ ). Similarly, the mean pressures to the thumb among FM participants were significantly lower than that of the healthy controls: mild pain (Mean \pm SD FM: $1.93 \pm 1.14 \mathrm{~kg}$, HCs: $3.64 \pm 2.63 \mathrm{~kg}, P=0.007$ ), slightly intense (Mean \pm SD FM: $3.16 \pm 1.69 \mathrm{~kg}$, HCs: $5.78 \pm 2.28 \mathrm{~kg}, P=0.0001)$ and numerically lower for faint pain stimuli (Mean \pm SD FM: $0.69 \pm 0.74 \mathrm{~kg}$, HCs: $0.29 \pm 0.16 \mathrm{~kg}, P=0.17$ ) (Figure 2).

\section{Analysis 1: VVD and FM participants display augmented brain in response to thumb pressure pain-Comparing VVD, FM, and HCs, greater activity at the left insular cortex was detected in both the pain groups as compared to pain free controls (Table I) (Figure 3A). A post hoc analysis demonstrated that VVD and FM both had greater activations in the insula than $\mathrm{HC}$ (Figure 3B), with an overlapping area of activity detected in the FM and VVD groups (Figure 3C).}

\section{Analysis 2: Augmented brain activation is present in VVD participants-VVD} patients also showed significantly increased brain activity in several pain regions during slightly intense pressure stimuli to the thumb as compared to $\operatorname{HC}\left(P_{\text {corrected }}<0.005\right)$. These regions included the insula, dorsal mid-cingulate, posterior cingulate and the ventral posterolateral nuclei of the thalamus (Figure 4 and Table I). Consistent with this finding we also saw greater activation in VVD patients compared to $\mathrm{HC}$ in other pressure pain 
conditions such as faint and mild pain in regions including the insula, posterior cingulate and thalamus (see Supplementary Figure 1).

In response to vulvar pressure stimulation, VVD patients did not display significantly altered brain activity compared to HCs in any region in the whole brain analysis. No greater deactivations were seen in VVD in this analysis.

\section{Analysis 3: Greater pressure pain brain activations observed in VVD as compared to $\mathrm{HC}$ in $\mathrm{ROI}$ analysis}

Thumb pressure stimulation: VVD patients show significantly greater activations compared to HCs in regions identified in other studies including: bilateral secondary somatosensory cortex, premotor cortex, anterior insula and mid insula $(P<0.05)$ (Figure 5A). Correlation analysis between SF-MPQ scores of VVD patients and activation patterns showed a significant positive correlation with primary somatosensory cortex found at the thumb region in the sensory homunculus map $(P=0.04 ; \mathrm{r}=0.44)$ (Figure 5B) and inferior parietal lobule $(P=0.02 ; \mathrm{r}=0.50)$ (Figure 5C).

Vulvar pressure stimulation: VVD patients showed significantly less deactivation compared to HCs in the inferior parietal lobule (IPL) region (Figure 5A), a task negative structure. No statistically significant correlation was seen between SF-MPQ scores and vulvar pressure stimulus activation.

\section{Analysis 4: Differential BOLD activity in response to pressure stimulation in proposed subgroups of VVD}

Thumb pressure stimulation: The characteristics of vulvar pain among our VVD participants in relation to previously proposed subgrouping are shown (Table 2). Two participants were not classified as either primary or secondary VVD pain. Thumb pressure ROIs from analysis 2 showed significantly higher brain activations in participants who reported primary VVD pain $(\mathrm{n}=14)$ compared to participants that reported secondary VVD pain $(n=8)$ (Figure 6A). No significant difference between primary and secondary groups was noted in other areas that showed elevated BOLD response in VVD (from Analysis 2). Significantly higher brain activity was also noted at the posterior cingulate region in the provoked-only pain participants $(n=14)$ compared to that of participants who also had unprovoked pain $(n=10)$ (Figure 6B) in response to thumb pressure stimuli. No significant difference was noted in other areas from Analysis 2 ROIs, when comparing those with provoked-only pain versus those with unprovoked pain.

No significant differences in brain activity were noted when comparing participants with generalized VVD pain versus localized pain.

Vulvar pressure stimulation: This analysis included participants that reported provokedonly vulvar pain versus participants that also experienced unprovoked pain. Pain-related activation during vulvar stimulation was significantly greater at the precuneus region in participants with provoked-only pain compared to those who reported unprovoked pain (Figure 7) (Peak voxel coordinates $\mathrm{x}, \mathrm{y}, \mathrm{z}=0,-68,60 ;$ Zscore=4.33; cluster size $=204 ; P<0.05$ corrected for multiple comparison). Individual BOLD responses confirmed this finding.

No significant differences were detected during the other subgroup comparisons including the generalized versus localized pain participants and the primary versus secondary VVD participants, in response to vulvar stimulation. 


\section{Discussion}

These findings suggest that some VVD patients may have significant alterations in brain processing of painful stimuli. As our previous work ${ }^{14}$ demonstrated that VVD patients have a global widespread reduction in pressure pain thresholds (i.e. generalized hyperalgesia), here we find greater sensitivity to suprathreshold experimental pain stimuli at a site distant from their region of clinical pain which is associated with significantly greater brain activation responses in several pain-responsive brain regions. These data suggest that the pathology in vulvodynia patients may involve regions other than the vulva. Interestingly, the augmented brain response seen in brain areas of VVD patients was also found in patients diagnosed with FM. Of significance, the insular cortex, a region commonly seen with augmented response in FM, displayed heightened overlapping activity in both VVD and FM patients. This suggests that these two chronic pain states may share a common neurobiological pathophysiology, however, as this was a cross-sectional examination, we cannot infer a causal relationship between our imaging findings and the presence of chronic pain.

The regions that showed greater activation differences between the VVD and HCs groups, such as the insula, posterior cingulate, thalamus and contralateral dorsal mid cingulated region, are among those commonly activated during pain stimulation. ${ }^{6,12} \mathrm{We}$ interpret these finding as evidence of additional brain regions associated with augmented pain processing in VVD. While these other areas are also known to play a role in pain processing, we find the area of greatest difference identified between the VVD participants and HCs was the ipsilateral insula. The insula is known to be a sensory integration region involved in pain and sensory processing. ${ }^{10,11}$ Interestingly, our ROI analysis, which was based on previously published fMRI studies in VVD and FM, also showed significant differences between VVD patients and $\mathrm{HC}$ in the premotor cortex, somatosensory and insula cortex in response to thumb pressure stimuli and inferior parietal lobule in response to vulvar pressure stimuli. Elevated brain activation within the primary somatosensory cortex and IPL were also associated with more clinical pain. Of note, the identified primary somatosensory region corresponded somatotopically to the thumb, which may contribute to the evidence that the VVD patients in this sample displayed generalized hyperalgesia.

As an exploratory analysis, we also found brain differences between previously suggested subgroups within the larger VVD cohort (Analysis 4). Primary onset VVD participants and provoked pain participants showed significantly greater brain activation compared to secondary onset and unprovoked pain participants, respectively, at the posterior cingulate region in response to thumb pressure stimuli. Similarly, in response to vulvar pressure stimuli in VVD participants, our provoked-only subgroup showed significantly greater activation compared to our unprovoked pain group in the precuneus region ${ }^{7}$. While the results are preliminary and potentially underpowered they nevertheless suggest VVD subgroups. The posterior cingulate and precuneus are regions previously shown to be part of the Default Mode Network (DMN), ${ }^{5}$ a constellation of brain regions involved in internal self-referential thinking and autobiographical memory. We recently reported that FM patients have enhanced intrinsic connectivity between the insula and regions of the DMN. ${ }^{27}$ Moreover, the degree of enhanced connectivity was associated with spontaneous clinical pain ratings in this population. We speculate that the enhanced pain evoked brain activity present in primary and provoked subgroups of VVD may be related to altered DMN activity in these patients. This in turn may be related to the different clinical expression of pain in these subgroups. We hypothesize that the reason for lower activation in those with unprovoked pain may be due to the fact that these patients experience pain much or all the time, leading to a state of chronic activation. Because our fMRI analyses compared pain to a rest condition, the unprovoked patient may have ongoing activity thus limiting the change in 
response observed when provoked pain is applied. The lack of evidence when comparing generalized versus localized pain in response to both thumb and vulvar pressure stimulation contributes to mounting evidence that the distinction between the generalized and localized VVD subgroups may not reflect physiologic differences. ${ }^{30}$

Overall, our results are consistent with other functional imaging studies that have suggested augmented brain processing in chronic pain conditions including FM, ${ }^{17,9}$ chronic low back pain ${ }^{15}$ and osteoarthritis ${ }^{18}$. In the only previous fMRI VVD study done using vulvar stimulation during functional neuroimaging, Pukall et al showed activity among VVD in the insula, somatosensory, premotor and dorsal anterior cingulate cortex regions. ${ }^{29}$ However no significant results were reported in Pukall's paper when directly comparing VVD patients and controls. Similarly, we did not observe significant differences in the brain response to pain induced by vulvar pressure stimuli in the whole brain analysis (Analysis 2). Further, Pukall examined provoked patients only, while our sample included those with provoked as well as those with unprovoked pain; also the stimulus pressure intensities used by Pukall were kept constant for both patients and healthy controls, but in our study we matched subjects on pain perception (faint, mild, slightly intense) by using pressures that produced similar levels of pain.

We acknowledge that our study has a number of limitations. First, since this study was cross sectional in design, we cannot say whether the augmented neural activity noted is a consequence of the pain condition or whether the augmented neural activity is driving the perceived pain (see above). Another limitation was that since our inter-stimulus intervals were non-variable, the resultant activations might be influenced by anticipatory effects, although this is unlikely given that the levels of pressures administered were randomized. Furthermore there was a 20\% exclusion or drop-out in the study. This could have resulted in a lack of generalizability of the study findings. We also cannot exclude the possibility that participants may have other or overlapping pain conditions, which may have been undiagnosed. Patients with known chronic pain conditions were excluded from the FM cohort while HCs may have had transient vulvar pain (less than 3 months) without being excluded. We excluded any patients reporting FM among the VVD patient cohort, and there were no reports of VVD among the FM patient cohort. However, we recognize that although these pain conditions may occur concurrently, they are often undiagnosed or underrecognized. Furthermore, our study involved a relatively small sample of patients, thus potentially limiting the generalizability of our findings. Future analysis with a larger population may allow more powerful assessments of inter-related CNS pain loci, which would further clarify the interaction of the multiple sites responsive to provoked pain.

Augmented central pain processing in this chronic pain cohort is supported by the findings of augmented brain reactivity as measured by fMRI scanning in participants suffering from VVD who were stimulated at a site remote from the vulva (the thumb), and by the correlation of these activations to their clinical pain. In addition, we present preliminary data supporting previously proposed VVD sub-groups, including those with primary versus secondary VVD and those with and without spontaneous (unprovoked) pain. These findings provide guidance for future subgroup analyses. Brain factors may need to be considered to maximize successful treatment of some VVD patients.

\section{Supplementary Material}

Refer to Web version on PubMed Central for supplementary material. 


\section{Acknowledgments}

This study was supported by grants from the National Institute of Child Health and Human Development of the National Institutes of Health (R01 HD045661) and Supplemental funding (R01 HD045661-S1) and a Department of Defense award W81XWH-07-02-0050. Dr. Harris was supported by a Dana Foundation Award in Brain and Immuno-imaging.

We would like to thank Eric Ichesco and Tobias Schmidt-Wilcke for contribution to the fMRI analysis, Sue Countryman for participant enrollment and baseline sensory testing, and Keith Newnham, our fMRI technician.

\section{References}

1. Bajaj P, Arendt-Nielsen L, Madsen H. Sensory changes during the ovulatory phase of the menstrual cycle in healthy women. Eur J Pain. 2001; 5:135-144. [PubMed: 11465979]

2. Bornstein J, Maman M, Abramovici H. "Primary" versus "secondary" vulvar vestibulitis: one disease, two variants. Am J Obstet Gynecol. 2001; 184:28-31. [PubMed: 11174475]

3. Brett, M.; Anton, J.; Valabregue, R.; Poline, J. Region of interest analysis using SPM toolbox.. Proceedings of e 8th International Conference on Functional Mapping of the Human Brain;

4. Bromm B. The involvement of the posterior cingulate gyrus in phasic pain processing of humans. Neurosci Lett. 2004; 361:245-249. [PubMed: 15135939]

5. Buckner RL, Andrews-Hanna JR, Schacter DL. The brain's default network: anatomy, function, and relevance to disease. Ann N Y Acad Sci. 2008; 1124:1-38. [PubMed: 18400922]

6. Casey KL. Match and mismatch: identifying the neuronal determinants of pain. Ann Intern Med. 1996; 124:995-998. [PubMed: 8624067]

7. Cavanna AE, Trimble MR. The precuneus: a review of its functional anatomy and behavioural correlates. Brain. 2006; 129:564-583. [PubMed: 16399806]

8. Coghill RC, Sang CN, Maisog JM, Iadarola MJ. Pain intensity processing within the human brain: a bilateral, distributed mechanism. J Neurophysiol. 1999; 82:1934-1943. [PubMed: 10515983]

9. Cook DB, Lange G, Ciccone DS, Liu WC, Steffener J, Natelson BH. Functional imaging of pain in patients with primary fibromyalgia. J Rheumatol. 2004; 31:364-378. [PubMed: 14760810]

10. Craig AD, Chen K, Bandy D, Reiman EM. Thermosensory activation of insular cortex. Nat Neurosci. 2000; 3:184-190. [PubMed: 10649575]

11. Craig AD. How do you feel--now? The anterior insula and human awareness. Nat Rev Neurosci. 2009; 10:59-70. [PubMed: 19096369]

12. Derbyshire SW. Imaging visceral pain. Curr Pain Headache Rep. 2007; 11:178-182. [PubMed: 17504644]

13. Foster DC, Dworkin RH, Wood RW. Effects of intradermal foot and forearm capsaicin injections in normal and vulvodynia-afflicted women. Pain. 2005; 117:128-136. [PubMed: 16087295]

14. Giesecke J, Reed BD, Haefner HK, Giesecke T, Clauw DJ, Gracely RH. Quantitative sensory testing in vulvodynia patients and increased peripheral pressure pain sensitivity. Obstet Gynecol. 2004; 104:126-133. [PubMed: 15229011]

15. Giesecke T, Gracely RH, Grant MA, Nachemson A, Petzke F, Williams DA, Clauw DJ. Evidence of augmented central pain processing in idiopathic chronic low back pain. Arthritis Rheum. 2004; 50:613-623. [PubMed: 14872506]

16. Gracely RH, Lota L, Walter DJ, Dubner R. A multiple random staircase method of psychophysical pain assessment. Pain. 1988; 32:55-63. [PubMed: 3340422]

17. Gracely RH, Petzke F, Wolf JM, Clauw DJ. Functional magnetic resonance imaging evidence of augmented pain processing in fibromyalgia. Arthritis Rheum. 2002; 46:1333-1343. [PubMed: 12115241]

18. Gwilym SE, Keltner JR, Warnaby CE, Carr AJ, Chizh B, Chessell I, Tracey I. Psychophysical and functional imaging evidence supporting the presence of central sensitization in a cohort of osteoarthritis patients. Arthritis Rheum. 2009; 61:1226-1234. [PubMed: 19714588]

19. Haefner HK, Collins ME, Davis GD, Edwards L, Foster DC, Hartmann ED, Kaufman RH, Lynch PJ, Margesson LJ, Moyal-Barracco M, Piper CK, Reed BD, Stewart EG, Wilkinson EJ. The vulvodynia guideline. J Low Genit Tract Dis. 2005; 9:40-51. [PubMed: 15870521] 
20. Harlow BL, Stewart EG. A population-based assessment of chronic unexplained vulvar pain: have we underestimated the prevalence of vulvodynia? J Am Med Womens Assoc. 2003; 58:82-88. [PubMed: 12744420]

21. Harris RE, Sundgren PC, Craig AD, Kirshenbaum E, Sen A, Napadow V, Clauw DJ. Elevated Insular Glutamate in Fibromyalgia Is Associated With Experimental Pain. Arthritis and Rheumatism. 2009; 60:3146-3152. [PubMed: 19790053]

22. Hofbauer RK, Rainville P, Duncan GH, Bushnell MC. Cortical representation of the sensory dimension of pain. J Neurophysiol. 2001; 86:402-411. [PubMed: 11431520]

23. Kuchinad A, Schweinhardt P, Seminowicz DA, Wood PB, Chizh BA, Bushnell MC. Accelerated brain gray matter loss in fibromyalgia patients: premature aging of the brain? J Neurosci. 2007; 27:4004-4007. [PubMed: 17428976]

24. Leclair CM, Goetsch MF, Korcheva VB, Anderson R, Peters D, Morgan TK. Differences in primary compared with secondary vestibulodynia by immunohistochemistry. Obstet Gynecol. 2011; 117:1307-1313. [PubMed: 21606740]

25. Melzack R. The short-form McGill Pain Questionnaire. Pain. 1987; 30:191-197. [PubMed: 3670870]

26. Moyal-Barracco M, Lynch PJ. 2003 ISSVD terminology and classification of vulvodynia: a historical perspective. J Reprod Med. 2004; 49:772-777. [PubMed: 15568398]

27. Napadow V, LaCount L, Park K, As-Sanie S, Clauw DJ, Harris RE. Intrinsic brain connectivity in fibromyalgia is associated with chronic pain intensity. Arthritis Rheum. 2010; 62:2545-2555. [PubMed: 20506181]

28. Petzke F, Harris RE, Williams DA, Clauw DJ, Gracely RH. Differences in unpleasantness induced by experimental pressure pain between patients with fibromyalgia and healthy controls. Eur J Pain. 2005; 9:325-335. [PubMed: 15862482]

29. Pukall CF, Strigo IA, Binik YM, Amsel R, Khalife S, Bushnell MC. Neural correlates of painful genital touch in women with vulvar vestibulitis syndrome. Pain. 2005; 115:118-127. [PubMed: 15836975]

30. Reed BD, Gorenflo DW, Haefner HK. Generalized vulvar dysesthesia vs vestibulodynia. Are they distinct diagnoses? J Reprod Med. 2003; 48:858-864. [PubMed: 14686018]

31. Reed BD, Haener HK, Sen A, Gorenflo DW. Vulvodynia incidence and remission rates among adult women - A 2-year follow-up study. Obstetrics and Gynecology. 2008; 112:231-237. [PubMed: 18669716]

32. Riley L 3rd, Robinson ME, Wise EA, Price DD. A meta-analytic review of pain perception across the menstrual cycle. Pain. 1999; 81:225-235. [PubMed: 10431710]

33. Silverman DH, Munakata JA, Ennes H, Mandelkern MA, Hoh CK, Mayer EA. Regional cerebral activity in normal and pathological perception of visceral pain. Gastroenterology. 1997; 112:64 72. [PubMed: 8978344]

34. Tracey I. Nociceptive processing in the human brain. Curr Opin Neurobiol. 2005; 15:478-487. [PubMed: 16019203]

35. Worsley KJ, Liao CH, Aston J, Petre V, Duncan GH, Morales F, Evans AC. A general statistical analysis for fMRI data. Neuroimage. 2002; 15:1-15. [PubMed: 11771969] 


\section{Perspective}

The presence of augmented brain responses to pressure stimuli remote from the vulva was observed in vulvodynia patients. These findings may guide treatment decisions for better response as brain mechanisms may be a factor in some VVD patients. 


\section{Total enrolled subjects}

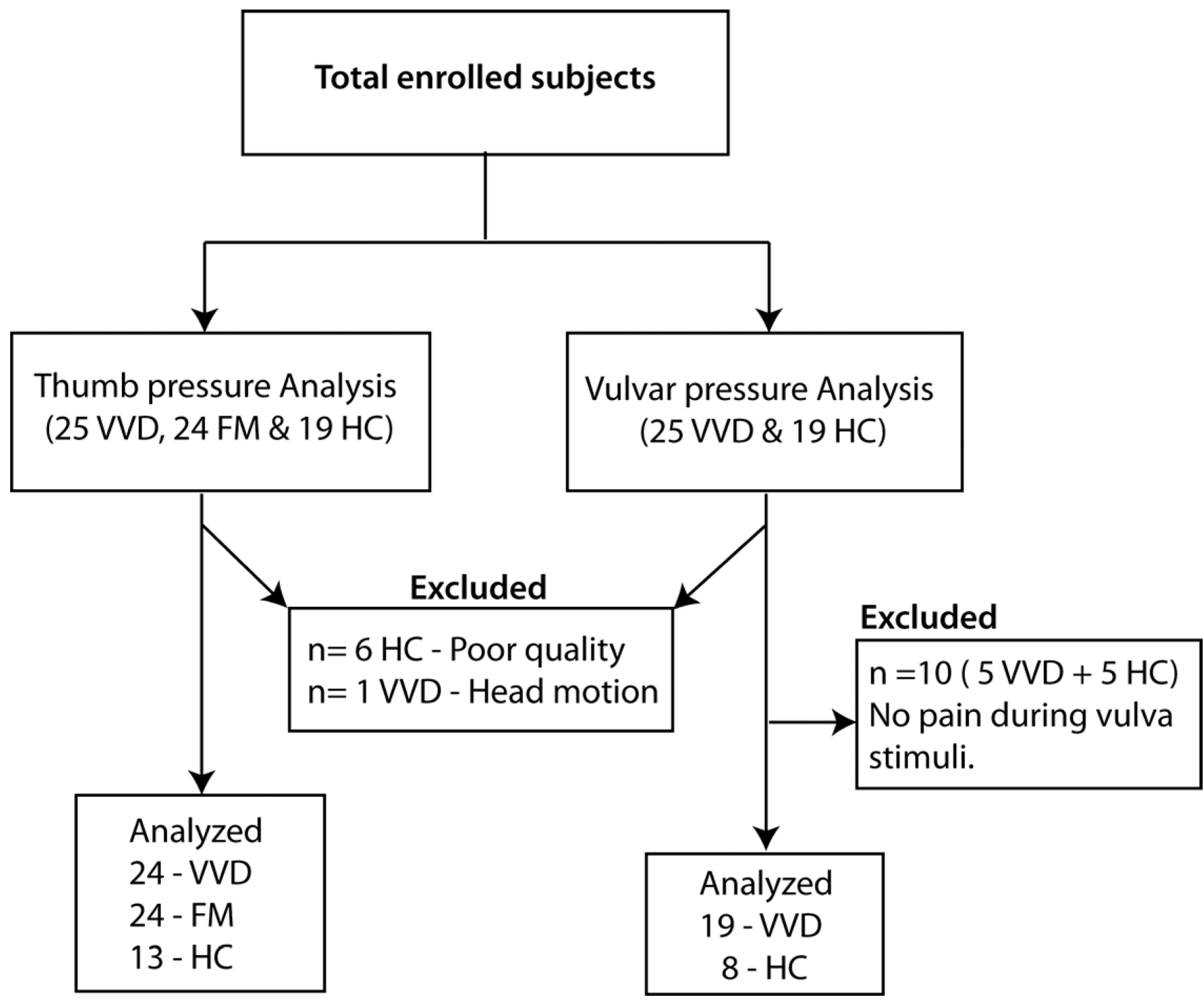

Figure 1. Diagram of Analyses and participant exclusion

Of the total subjects enrolled in the study, $24 \mathrm{VVD}, 24 \mathrm{FM}$ and $13 \mathrm{HC}$ were included in the thumb pressure stimuli analysis. The Vulvar pressure stimuli analysis was done on 19 VVD and $8 \mathrm{HC}$. 
A)

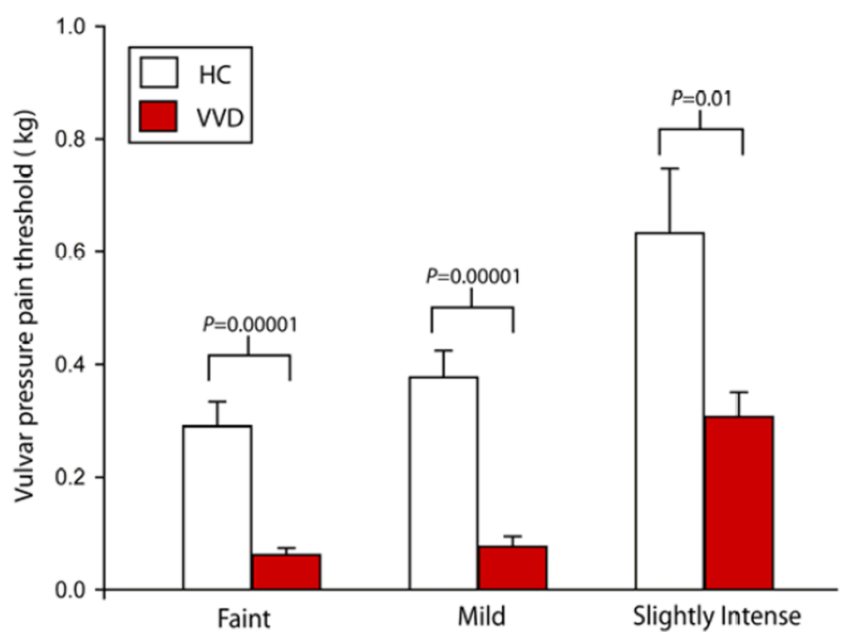

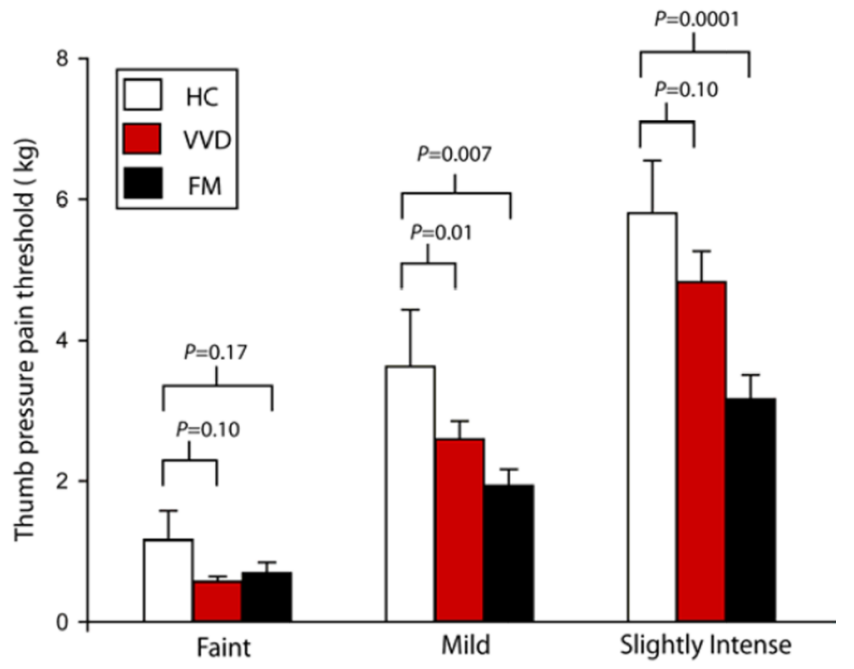

Figure 2. VVD and FM patients display lower pressure pain thresholds

Bar graphs of A) Mean and standard error of vulvar pressure pain thresholds among $\mathrm{HC}$ (white) and VVD patients (red). VVD patients had significantly lower pain threshold compared to HCs. B) mean and standard error of thumb pressure pain thresholds among HC (white), VVD (red) and FM (black) participants. VVD and FM displayed lower pain thresholds compared to $\mathrm{HC}$. 
(A)

(C)
(B)

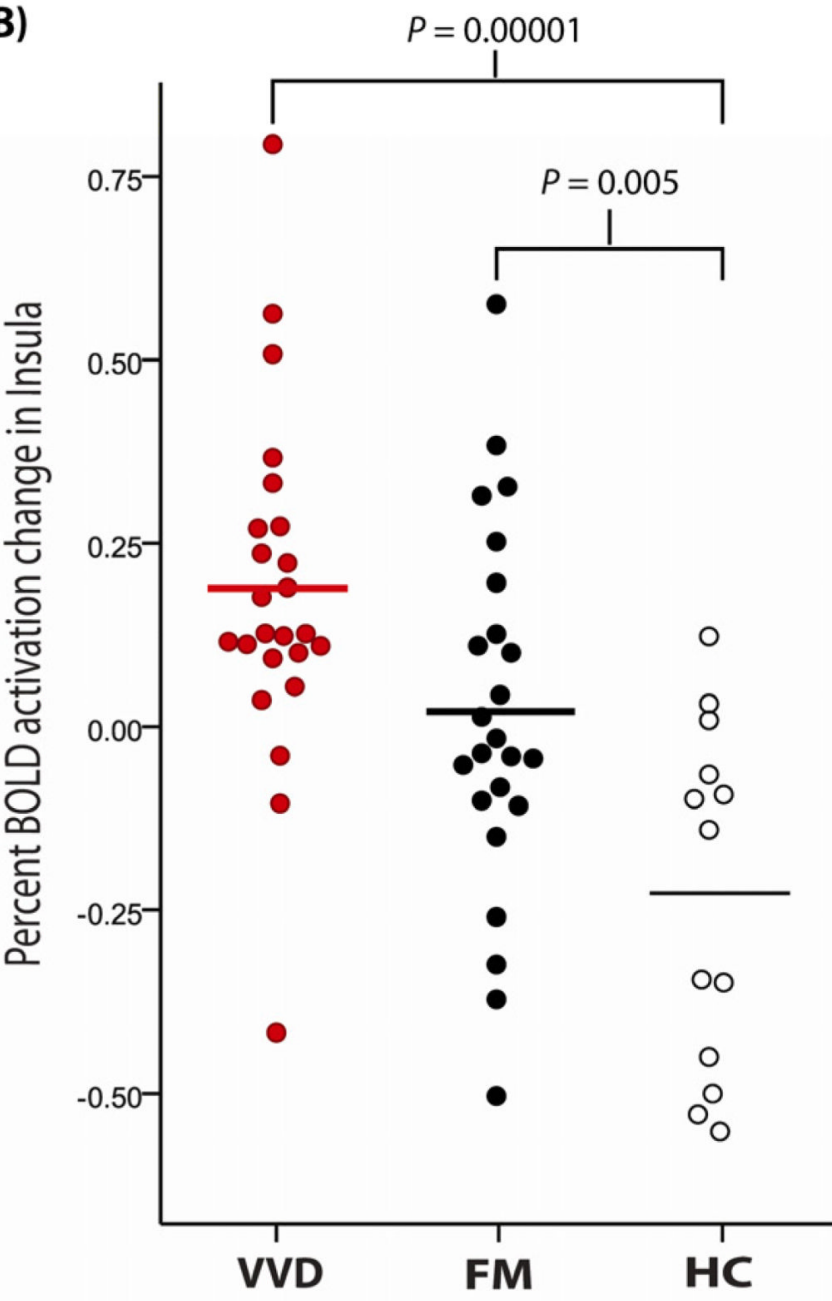

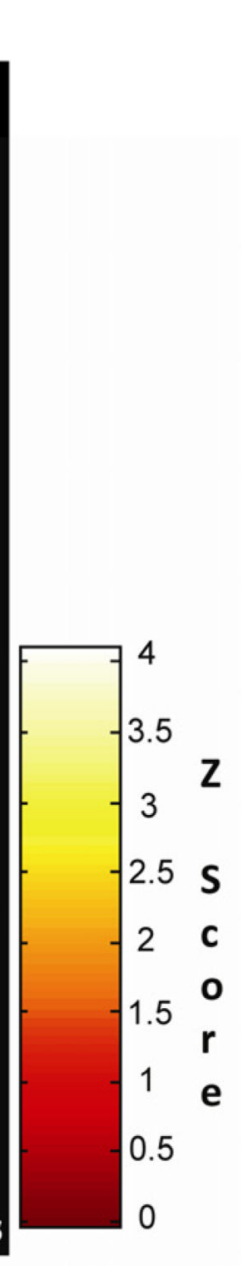

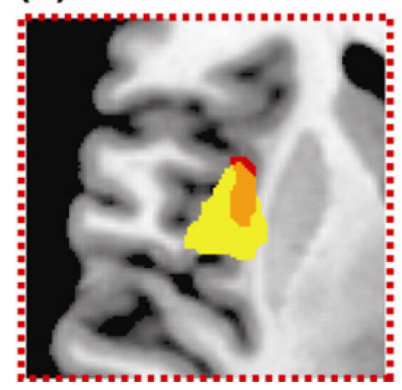

Figure 3. VVD and FM participants show similar enhanced insular activation Analysis of VVD, FM and HCs, (A) shows greater activations at the insula in both the pain groups. The region of significant difference in activation is shown in standard space superimposed on a structural T1-weighted magnetic resonance image. Image is shown in neurological view, with the right side of the brain on the right. (B) Scatter plot depicts individual BOLD activations at the insula region in individuals with VVD (red), FM (black) and HCs (white) with their P values from post hoc ANOVA analysis. (C) Shows the identified insula cluster in both the contrast conditions, VVD minus HCs (Red) and FM minus HCs (yellow). Orange shows common region of activation between VVD and FM. For viewing, the voxel level $P$ threshold was set at 0.005 . 

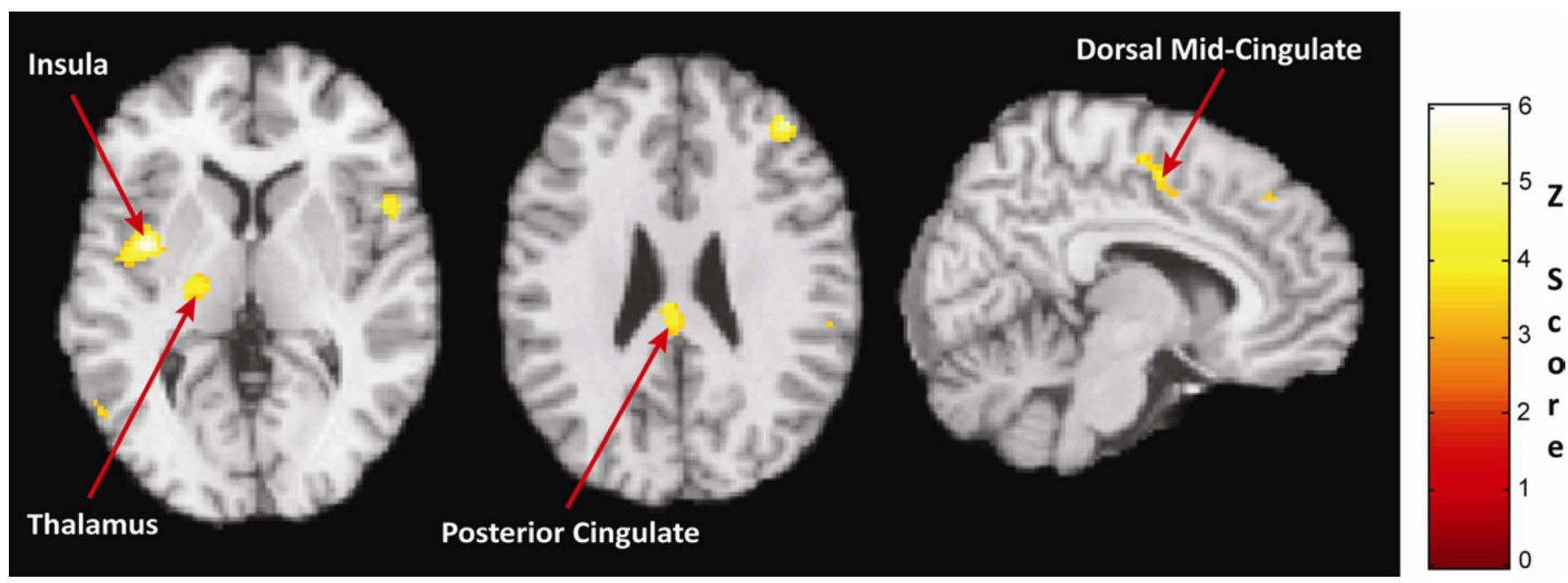

Figure 4. Augmented brain activity in VVD patients

Pain activation in response to thumb pressure stimulus in VVD participants and pain-free HCs. Regions of significant difference in activation are shown in standard space superimposed on a structural T1-weighted magnetic resonance image. The regions of activations in the brain are marked in yellow with their corresponding $\mathrm{Z}$ score intensity map. Images are shown in neurological view. 


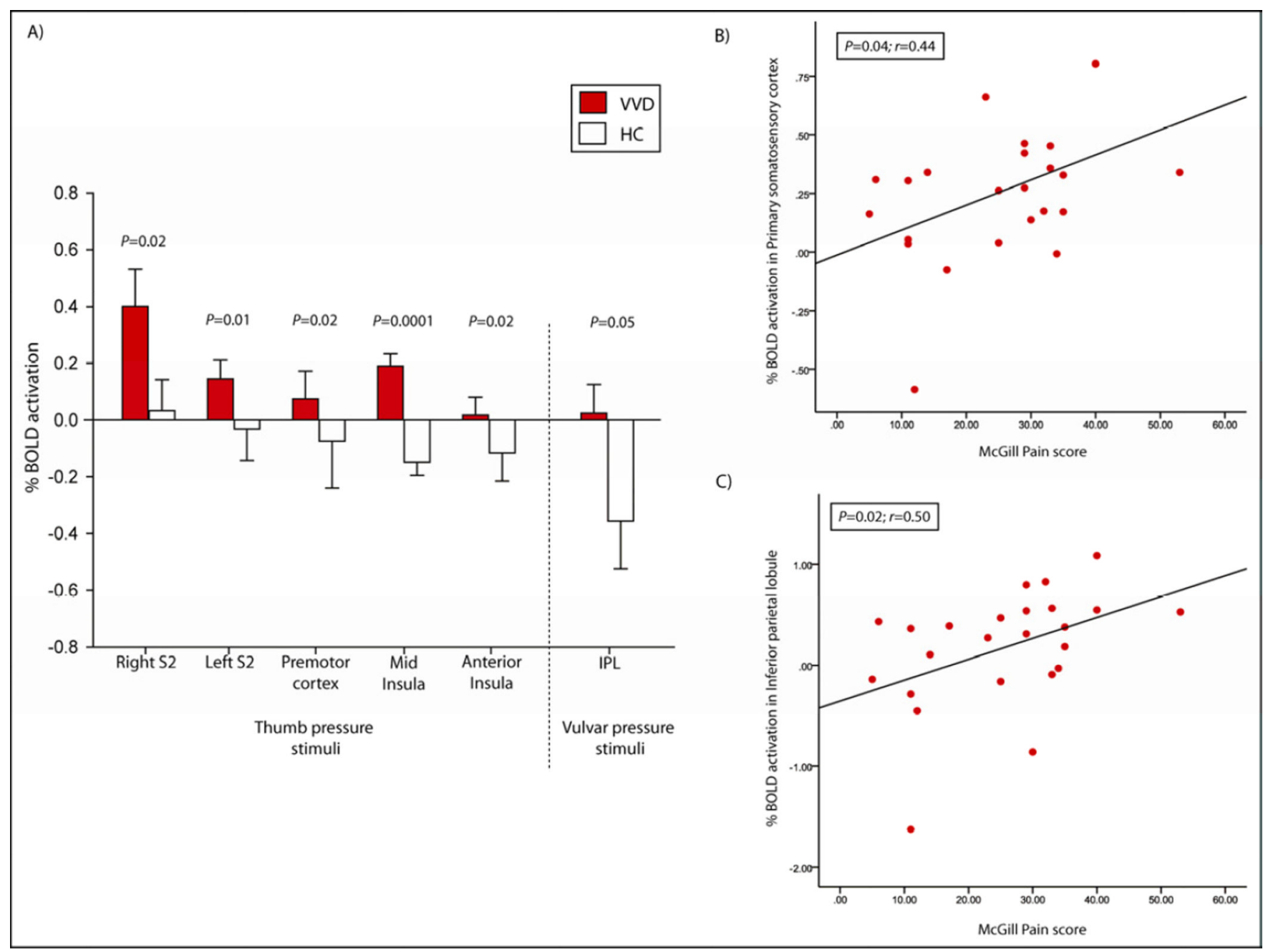

Figure 5. VVD patients display increased brain activity to thumb and vulvar pressures in regions previously identified

A) bar graphs of mean and standard error shows greater percent BOLD activations in VVD patients (red) as compared to controls (white) in response to both thumb and vulvar pressure stimulation. Scatter plots show significant correlations between clinical pain scores and percent BOLD activations within the $(\mathrm{B})$ primary somatosensory and $(\mathrm{C})$ inferior parietal lobule. 


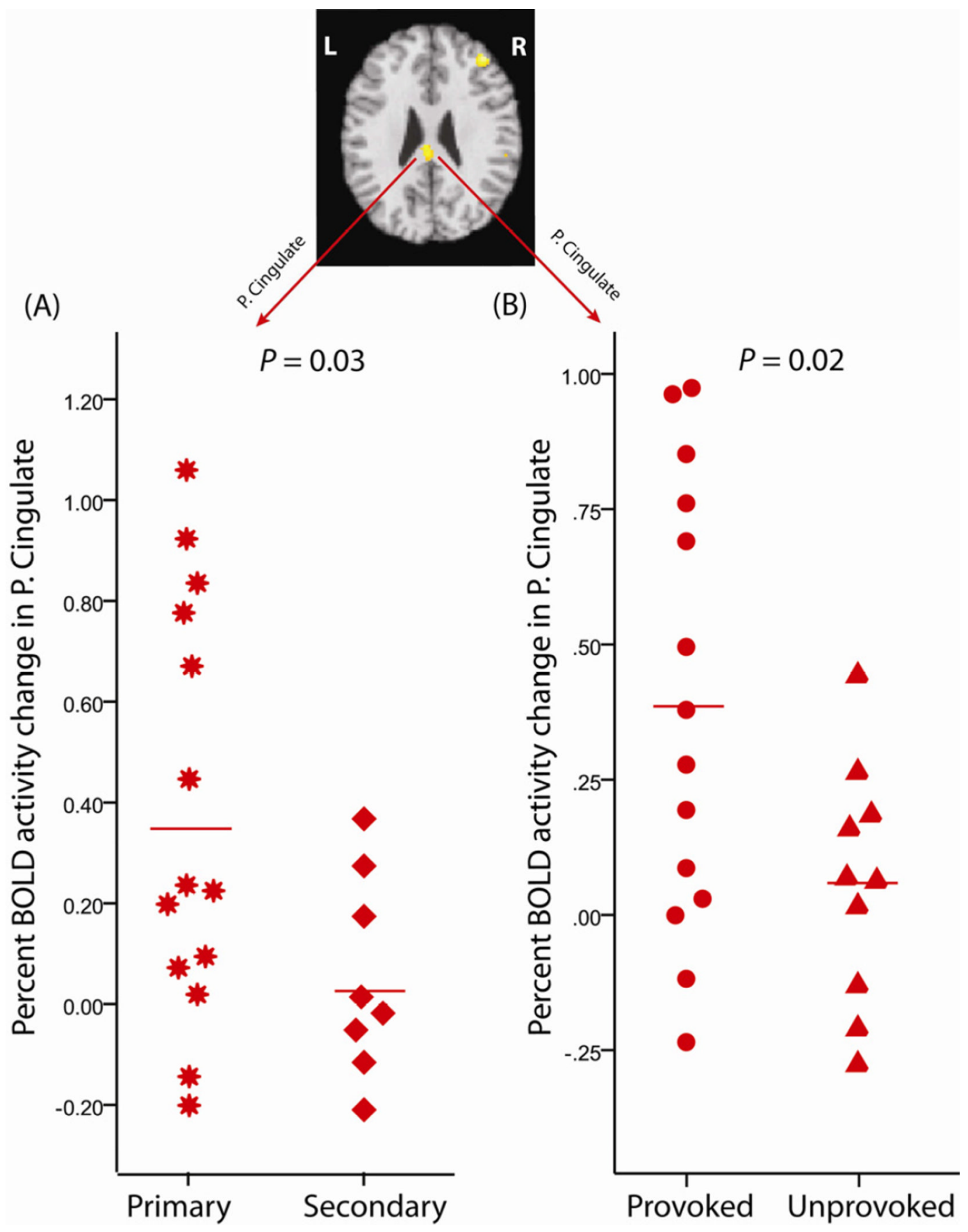

Figure 6. Differing VVD subgroups confirmed in response to thumb pressure stimuli The Posterior cingulate ROI identified in Analysis 2 showed greater activation in (A) primary (star) VVD participants $(\mathrm{n}=14)$ compared to secondary (diamond) VVD participants $(\mathrm{n}=8)$, and (B) Greater activation in VVD participants with pain that was provokable-only (circles) $(n=14)$ as compared to those who also reported unprovoked (triangles) pain $(n=10)$. 
(A)
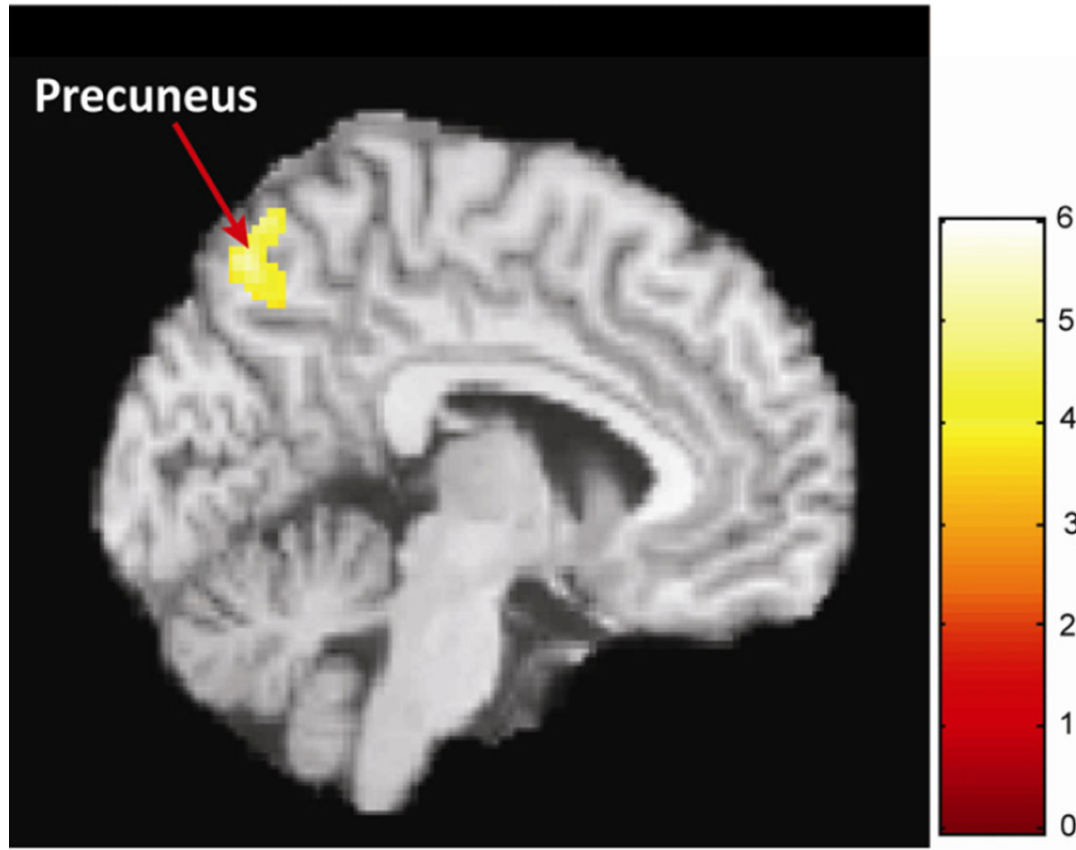

Figure 7. Differing VVD subgroups confirmed in response to vulvar pressure
(B)

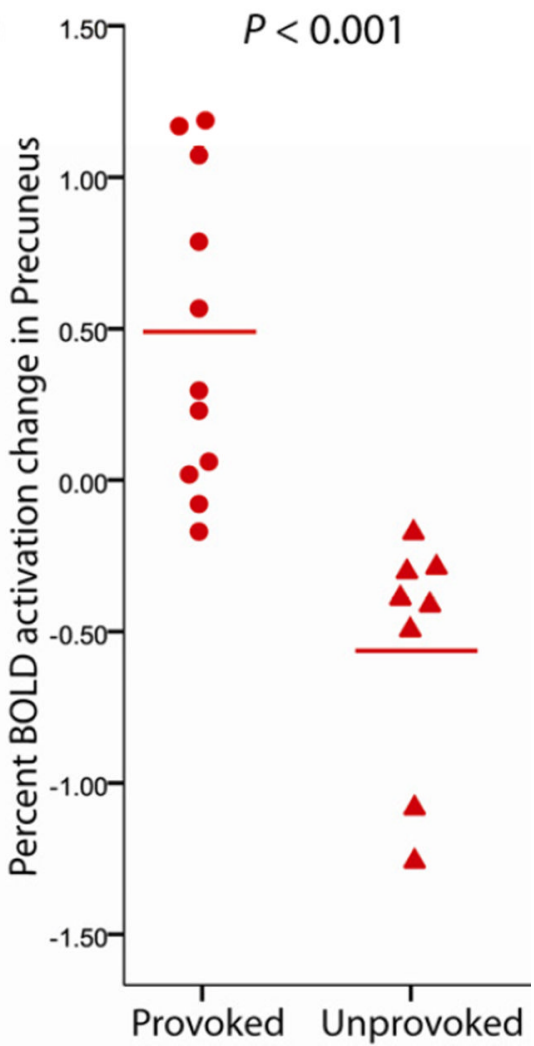
Greater vulvar pressure pain activations were observed during vulvar stimulation among participants with provokable-only (circles) pain $(n=11)$ compared to those who also reported unprovoked (triangles) pain $(n=8)$. (A) Sagittal slice shows precuneus region activation in yellow ( $\mathrm{P}<0.05$ corrected for multiple comparison). (B) Scatter plot depicts percent change in BOLD activation at the precuneus region when these two groups were compared. 


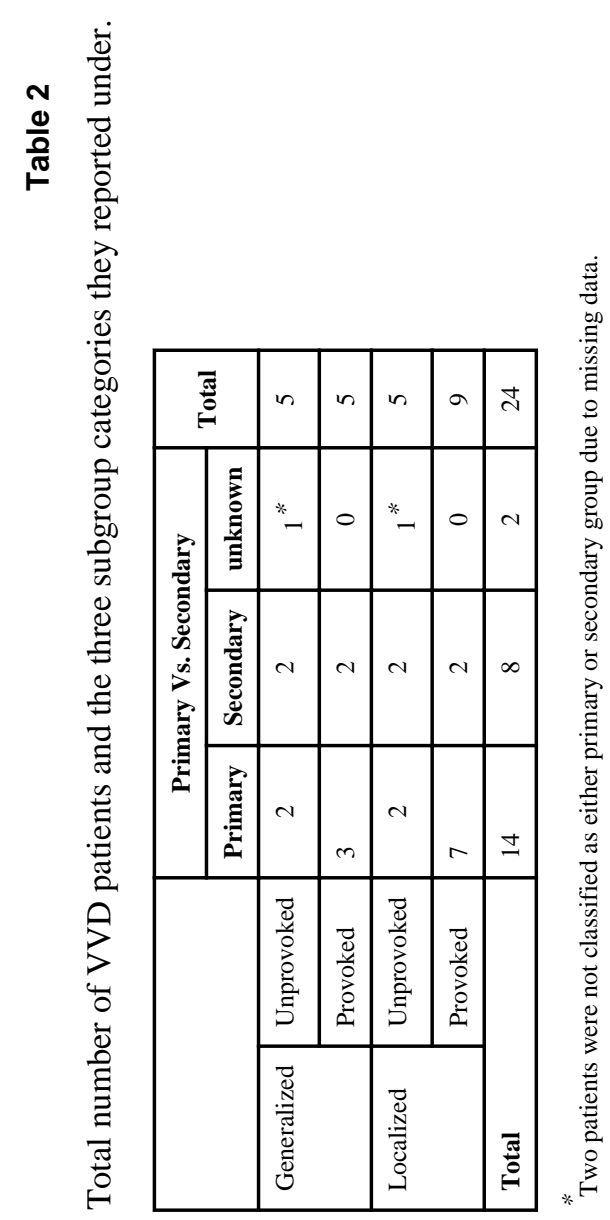

J Pain. Author manuscript; available in PMC 2014 June 01. 